\title{
Screen-Printed Graphite Nanoplate Conductive Ink for Machine Learning Enabled Wireless Radiofrequency- Identification Sensors
}

DOI:

10.1021/acsanm.9b01034

\section{Document Version}

Accepted author manuscript

Link to publication record in Manchester Research Explorer

Citation for published version (APA):

Leng, T., Pan, K., Zhang, Y., Li, J., Afroj, S., Novoselov, K., \& Hu, Z. (2019). Screen-Printed Graphite Nanoplate Conductive Ink for Machine Learning Enabled Wireless Radiofrequency-Identification Sensors. ACS Applied Nano Materials. https://doi.org/10.1021/acsanm.9b01034

\section{Published in:}

ACS Applied Nano Materials

\section{Citing this paper}

Please note that where the full-text provided on Manchester Research Explorer is the Author Accepted Manuscript or Proof version this may differ from the final Published version. If citing, it is advised that you check and use the publisher's definitive version.

\section{General rights}

Copyright and moral rights for the publications made accessible in the Research Explorer are retained by the authors and/or other copyright owners and it is a condition of accessing publications that users recognise and abide by the legal requirements associated with these rights.

\section{Takedown policy}

If you believe that this document breaches copyright please refer to the University of Manchester's Takedown Procedures [http://man.ac.uk/04Y6Bo] or contact uml.scholarlycommunications@manchester.ac.uk providing relevant details, so we can investigate your claim.

\section{OPEN ACCESS}




\section{Screen-Printed Graphite Nanoplate Conductive}

\section{Ink for Machine Learning Enabled Wireless}

\section{Radio-Frequency-Identification Sensors}

Ting Leng ${ }^{\dagger}$, Kewen Pan ${ }^{\dagger}$, Yongwei Zhang ${ }^{\dagger}$, Jiashen Lit, Shaila Afroj ${ }^{\S, ",}$, Kostya S. Novoselov

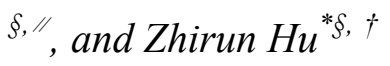

$\dagger$ School of Electrical and Electronic Engineering, University of Manchester, Manchester

$$
\text { M13 9PL, U.K }
$$

\#School of Materials, University of Manchester, Manchester M13 9PL, U.K

§National Graphene Institute, Manchester M13 9PL, UK.

// School of Physics and Astronomy, University of Manchester, Manchester M13 9PL, UK.

*Email: z.hu@manchester.ac.uk 
ABSTRACT In this study, we demonstrate sustainable conductive screen printing ink containing graphite nanoplates (GNP) prepared by means of combining shear and ultrasonication exfoliation processes with the aid of mixed-solvent strategy of isopropanol (IPA)-water mixtures. The screen printed GNP ink features high conductivity of $4.66 \times$ $10^{4} \mathrm{~S} / \mathrm{m}$, green and low manufacturing cost and has the potential for industrial scale-up productions. We first benchmark the GNP conductive ink and screen printed samples to investigate the rheology of the ink and its compatibility for industrial production. The high loading GNP ink is further applied to scalable and efficient production of Ultra high frequency (UHF) Radio Frequency Identification (RFID) tag antenna and reader antenna. A low-cost, accurate wireless liquid sensing system capable of sensing the content of the liquids via machine learning datasets by feedforward neural network is demonstrated for ubiquitous IoT sensing applications. The experimental results demonstrate that the GNP ink printed RFID tags and reader antennas can provide satisfactory read range and performance for many practical RFID applications, such as inventory and security. Furthermore, the printed RFID wireless sensor incorporating neural network machine learning can accurately label the content of colorless, transparent and unidentified liquids, illustrating the potential in low-cost, green and industrial scalable production of GNP conductive ink printed RFID antennas and sensing systems for massive IoT applications deployment enabling ubiquitous wireless connectivity.

KEYWORDS: graphite nanoplates, GNP, conductive ink, screen printing, RFID, machine learning, solvent sensing 


\section{Introduction}

The potential to apply graphene's outstanding electronic, thermal, optical and mechanical properties into applications has been intensively studied in the recent years ${ }^{1}$. Despite the extensive potential applications, the primarily concerns in implementation of graphene and other 2-D materials into practical applications is currently limited due to the limitations of large-scale productions ${ }^{2}$. Consequently, a great deal of attention from academic and industrial researchers has been paid to developing cheaper, simpler and more scalable production methods for graphene from the laboratory to industrial scales ${ }^{3}$. For industrial scale applications such as mass production of conductive inks which do not necessarily require high quality monolayer or even few-layered graphene and a strict aspect ratio (lateral size to thickness ratio), the ink can be prepared with graphite nanoplates (GNP) on kilogram scale to increase the production efficiency ${ }^{2,4}$. The traditional printed electronics use subtractive metal etching, wasting substantial raw materials and producing chemical wastes which damage the environment ${ }^{5}$. In industrial scale production such wastes must be properly disposed which further increases the fabrication costs. Researchers have been investigating and exploring the feasibility of using printing techniques such as screen printing and inkjet printing to reduce the processing steps and overall costs in the recent years ${ }^{6}$. Inkjet printing has high resolutions but the multilayer printing steps and costs in large batches are not costeffective for mass production. Furthermore, metallic nanoparticle conductive inks often use noble metal such as gold or silver leading to high costs in mass production. Although there are cheaper options such as aluminum and copper nanoparticles, they tend to be oxidized easily when exposed, which not only limits the operating environment but also degrades the 
performance $^{7}$. The development of screen printed graphene techniques has enabled production of graphene-based antennas ${ }^{8,9}$, RFIDs $^{10}$, passive components ${ }^{11}$ and wearable electronics ${ }^{9}$ recently.

Screen printing is popular and matured technology as it adds simplicity, affordability, speed and adaptability to the fabrication process $^{7}$. Screen printing technique is more compatible with the high-viscosity inks ( $\mu \sim 0.5-5$ Pas) as it helps with the amount of the ink dispense out of the mesh and transfer to the substrate during screen printing process also to minimize ink flow on the substrate after the ink transfer ${ }^{7,12}$. Ideally, a distribution of different flake sizes should be used for high conductivity. Large lateral size flakes with a few tens of nanometer thickness can reduce the number of interfaces between the flakes, but do not guarantee good stacking and can result in a number of voids when printed. Thin and small flakes allow for the best stacking, however, they end up with the maximum number of interfaces resulting in higher contact resistance, which potentially can increase the overall resistance. With a hierarchical structure of the printed ink where the large flakes provide the backbone and the smaller flakes fill in the voids, high conductivity for printed electronic circuit applications could be achieved. The optimal distribution (so to achieve high conductivity) depends on many parameters, including the contact resistance between the flakes, the lateral and vertical sizes and conductivity of the individual flakes ${ }^{13}$.

It is worth to notice that carbon nanotubes (CNTs) have recently proved to be promising materials for applications such as electrically conductive fillers in heat dissipation devices, flexible and transparent conductive films and lightweight conducting wires/cables, etc. However, screen printing is an uncontrolled ink distribution process which introduces misalignment and many contacts among CNT assemblies during the printing. Such lack of 
distribution process control makes CNTs incompatible to be used with screen printing ${ }^{14,15}$. On the other hand, the GNP can be stacked to form thick, flexible layers using the compression technique ${ }^{8-11,13}$, allowing cheap and easy production of printed electronics with good mechanical and electrical properties.

Despite the potential in replacing metal printed circuits and antennas with printed graphene ones, the solvents used frequently to prepare printable graphene conductive ink such as N-methyl-2-pyrrolidone (NMP) and dimethylformamide (DMF) have high boiling points and are toxic ${ }^{16,17}$. Non-toxic solvent Cyrene (Dihydrolevoglucosenone) for graphene conductive ink has been reported recently ${ }^{13,18}$ but it is still of high boiling point of $227^{\circ} \mathrm{C}$ and remain at high price and these still hinder the graphene printed electronics applications ${ }^{2,13}$. A mixed-solvent strategy has been proposed for facile and green preparation of dispersions containing multi-layer graphene in water-IPA mixtures ${ }^{19,20}$, along with low-temperature preservation to prevent agglomeration for storage and transport purposes. However, conductive ink by water-IPA liquid phase exfoliation has so far been made by redispersing exfoliated graphene flakes in toxic and high boiling point solvent such as $\mathrm{NMP}^{21}$. In this work, a simple one-pot synthesized water-IPA GNP ink without solvent exchange has been proposed, investigated and characterized, revealing that it can be a promising candidate for industrial scale GNP conductive ink production. Furthermore, screen printed RFID antennas and their wireless sensing applications have been proposed and demonstrated experimentally.

Compared to inkjet printing ink, efficient and green production of screen printable high viscosity GNP/graphene ink are less well developed due to the high loading for the required viscosity and the typical ink volume for the production process where minimal solutionprocessed dispersions can be used directly ${ }^{4}$. A high loading viscosity ink is also devoid of the 
coffee ring effect and capable of uniform deposition of the ink, forming rectangular printed structures, resulting in a better inter-flake percolation, and hence a higher electrical conductivity $^{4,22}$. Recently, a water-phase, non-dispersion exfoliation method to produce highly crystalline graphene flakes in the form of concentrated slurries (5 wt\%) with high production efficiencies $(82 \sim 170 \mathrm{~g} / \mathrm{h})$ is reported ${ }^{23}$. However, due to the high ionic strength of the solution, this method tends to form low-viscosity dispersion, hence not suitable for screen printing process. Screen printing of high-resolution pristine graphene patterning using a silicon stencil has been reported ${ }^{24}$. However, the ink preparation requires repeated centrifugation and sonication as well as a high temperature at $400{ }^{\circ} \mathrm{C}$ to yield the grapheneethyl cellulose powder for the ink production. Furthermore, the silicon stencil and PDMS spacer require more processing time and steps for the fine resolution print hence not suitable for low cost industrial scale production. A high yield exfoliation (100\%) of graphite into few layer graphene flakes in aqueous solutions under high shear rate turbulent flow conditions has also been reported ${ }^{25}$. The conductivity of the prepared ink can reach $\sigma \sim 2 \times 10^{4} \mathrm{~S} / \mathrm{m}$ after thermal annealing at $300{ }^{\circ} \mathrm{C}$. However this is not suitable for printing patterns on paper substrates. A mild heating process on the gelation of graphene/binder dispersions has been presented $^{26}$. The graphene paste for screen printing is prepared by high shear mixing with solvent exchange but its conductivity is relatively low $\left(\sigma \sim 1.3 \times 10^{4}\right)$ due to the added binders ${ }^{26}$. This binder based graphene paste is further processed with photonic annealing and subsequent compression rolling, reaching a conductivity of $2.86 \times 10^{4} \mathrm{~S} / \mathrm{m}$ normalized to $25 \mu \mathrm{m}$ thickness ${ }^{27}$. It is also reported that by using nitrocellulose as the binder, an electrical conductivity of up to $4 \times 10^{4} \mathrm{~S} / \mathrm{m}$ could be achieved after binder decomposition annealing at $350{ }^{\circ} \mathrm{C}^{28}$. A high intensity xenon lamp was further introduced to effectively decompose 
ethyl cellulose without damaging polymeric substrates or the printed graphene, and retain a high electrical conductivity of $2.5 \times 10^{4} \mathrm{~S} / \mathrm{m}^{29}$. However the photonic annealing is not suitable for industrial production. For practical applications such as RFID antennas or other electronic components which require the transport of significant currents, the sheet resistance requirement should be as low as possible and a substantial thickness of the printed layer is not desirable for printed electronics ${ }^{4}$. Higher conductivity ink is preferable because printing with thinner thickness reduces the printing difficulty ${ }^{30}$. Furthermore, high temperature annealing raises challenges in industrial scale production and it also limits the choices of substrates ${ }^{4}$.

In this study, we use highly conductive screen printing ink with high loading of GNP produced through shear mixing followed by ultrasonic liquid phase exfoliation by using nontoxic, low boiling point and green mixture solvent of water-IPA. This work demonstrates that water-IPA can provide efficient exfoliation of GNP in a large loading for up-scaling production of carbon based conductive ink and the one-pot synthesis that does not require solvent exchange. The as-prepared ink can be further concentrated to high concentration of $50 \mathrm{mg} / \mathrm{mL}$ viscous screen printable ink. The GNP flake characterization, the inter-flake percolation, and printing compatibility have been studied. The printed samples only require mild temperature drying at $80{ }^{\circ} \mathrm{C}$. Conductivity as high as $4.66 \times 10^{4} \mathrm{~S} / \mathrm{m}$ can be achieved with the prepared samples with hydraulic compression.

UHF RFID technology operating at 865-868 and 902-928 MHz range has already been used widely in inventory tracking and security, it also shows the potential to be used as wireless sensors ${ }^{31}$. A typical UHF RFID system applies the principle of modulated backscatter signal from a passive tag antenna to transfer data back to the reader antenna. The 
application of RFID technology is growing continuously thanks to its contactless communication capability ${ }^{32,33}$. Screen printed UHF RFID tag and reader antennas are presented in this work as a proof of concept to demonstrate the compatibility of the GNP conductive ink with screen printing technique for wireless RFID sensing and communication applications. The read range of the GNP printed UHF RFID tag antenna, gain and the radiation pattern of the reader antenna reported here showcase that the GNP ink printed antennas can perform well for many practical applications, such as inventory and personnel tracking. As an application example, we present here a GNP ink printed machine learning enabled wireless RFID sensor, which is capable of accurately identify the content of different transparent colorless liquids through neural network machine learning, illustrating the potential of GNP conductive ink printed RFID antenna and wireless sensing system for low-cost, disposable, ubiquitous wireless connectivity and massive IoT applications.

\section{Experimental}

\subsection{Preparation of high loading GNP ink}

$4 \mathrm{~g}$ of graphite flakes (Alfa Aesar) were slowly added to IPA-water mixture (400 mL) containing 40 vol\% IPA whilst being shear mixed at $8000 \mathrm{rpm}$ using Silverson L4R mixer equipped with square hole high shear screen. The shear mixing is kept on for $2 \mathrm{~h}$, a glass water cooling cylinder keeps the temperature around $20{ }^{\circ} \mathrm{C}$. The dispersion then is transferred into glass bottle and bath ultrasonicated for $10 \mathrm{hr}$. The supernatant was carefully removed after centrifuged for $15 \mathrm{~min}$ at $500 \mathrm{rpm}$ twice to remove any thick and large flakes. The resulting dispersion was first filtered via a 300-mesh stainless steel screen then vacuum 
filtered with Whatman filter paper grade 5 to remove the excess IPA-water, leaving fluid paste-like ink with concentration of $50 \mathrm{mg} / \mathrm{mL}$.

\subsection{GNP Sample Characterizations}

To characterize the properties of the GNP, the supernatant collected after centrifugation, is spin-coated and dried on cleaned $\mathrm{Si} / \mathrm{SiO}_{2}$ substrates. Atomic force microscopy (AFM) is performed on samples using Bruker FastScan to examine the flake structure of individual flakes and flake thickness distribution. Scanning electron microscopy (SEM) was carried out using Zeiss Ultra 100 to characterize the lateral size of the GNP and GNP cross-section to visualize the thickness of the printed layers after compression. For Raman measurement the samples are characterized by Horiba XploRA PLUS. The conductivity of the samples prepared by the ink with different processing times are characterized by

$$
\sigma=\frac{1}{R_{S} \times t}
$$

where $R_{s}$ is sheet resistance, and $t$ is thickness of the printed sample. For the sheet resistance measurement, $100 \mu \mathrm{L}$ of the supernatants after 5 min low-speed centrifugation (500 rpm, 5 min) were collected for samples with various processing time, and dropped on filter paper (Whatman qualitative filter paper, Grade 5). The filter paper was held on a glass funnel (140 $\mathrm{mL}$ Aldrich Buchner) by vacuum to avoid coffee-ring effect. The ink was further annealed in an oven for $2 \mathrm{hr}\left(80^{\circ} \mathrm{C}\right)$ and compressed using a hydraulic compressor. The sheet resistance is obtained by measuring 5 printed samples using 4- point probe station (Jandel, RM3000) and semiconductor characterization system (Keithley, 4200C). The thickness is obtained by 
using average thickness of the printed GNP by cross-sectional SEM view of the compressed samples. The viscosity of the concentrated ink is measured with TRIOS Rheometer at $25^{\circ} \mathrm{C}$.

\subsection{Screen Printing of GNP conductive ink}

Screen printing was performed using semiautomatic pneumatics screen printer with a $70^{\circ}$ angle squeegee, at a printing speed of $50-150 \mathrm{~mm} \mathrm{~s}^{-1}$. Mesh size 24 (thread number per inch) was used. The electronic components, eg, antennas, were printed on printing paper substrate (Xerox Colotech A4 paper 250 gsm). After screen printing, the printed samples were dried at $80{ }^{\circ} \mathrm{C}$ for $4 \mathrm{hr}$ in the laboratory oven.

\section{Results and discussion}

\subsection{Characterization of GNP}

Firstly, the thickness, lateral size and the quality of GNP in the dispersion are investigated since these parameters govern the electrical and mechanical performance of the conductive ink. Two-steps centrifugation was used to sort the as-prepared dispersion with wide and different size fractions. The lateral size distribution histograms are shown in Figure 1a. It is observed that the majority of the GNP flakes are of the sizes of 2 to $4 \mu \mathrm{m}$, with a smaller size (multi-layer graphene flakes) tail of the distribution. The sizes of GNP flakes are crucially significant in influencing the flake alignment and electrical conductivity of the printed layers, which will determine the overall resistance of the ink and RF performance when they are screen printed into patterns and connected to IC chips ${ }^{13}$. Large flake sizes can reduce the number of interfaces between the flakes but may not stack well between the layers 
and produce voids. On the other hand, small flakes can have better stacking and fewer voids but more interfaces.

Raman spectroscopy of exfoliated GNP is shown in Figure 1b, illustrating the intensity associated with in-phase vibrations of the graphite lattice at D-band $(1354 \mathrm{~cm}-1)$ and G-band $(1578 \mathrm{~cm}-1)$. The intensity of D-band peak is much weaker than G-band peak, indicating fewer structural defects and edge disturbance of the GNP which is significant for electron flow. The broader 2D band compared to graphene distinguishes to be $\mathrm{GNP}^{34}$. AFM analysis on individual flakes (12 hr processing time) are shown in Figure 1c, it can be seen that small flakes are characterized with fewer layers (of thickness less than 10nm) whereas larger flakes have more layers, with average height of $\sim 30 \mathrm{~nm}$. It can be observed that large flakes have much uneven surface than that of smaller ones. This is because large graphite flakes tend to fragment rather than exfoliate because total attractive force increased linearly with the area of stacked graphite ${ }^{35}$. In the case of smaller flakes, delamination of each layer easily occurs. For this very reason, $2 \mathrm{hr}$ shear mixing treatment was used to reduce the flake size to avoid long hours of ultrasonication. The delayed sonication is to better exfoliate the graphite into GNP. The exfoliation efficiency using a shear mixer will reduce over time because the rotational speed and the rotor-stator gap are fixed. It is energy inefficient and time consuming to exfoliate the GNP down to mono or multi-layer graphene for low cost wireless sensing IoT applications, longer processing time not only increases fabrication costs but also causes significant surface defects and smaller lateral size flakes which degrade the electrical conductivity $^{13}$ (see Raman spectroscopy for small flakes in Supporting Information Figure S1). Thickness distribution histograms is shown in Figure 1d. It can be seen around 25\% of the small flakes lie in the thickness range less than $5 \mathrm{~nm}$ and $30 \%$ flakes have thickness 
between $5-10 \mathrm{~nm}$. The AFM image is provided in Supporting Information Figure S2. Thin and small flakes will end up with more number of interfaces, which can potentially increase the resistance ${ }^{13,36,37}$. Carefully control of exfoliation processes and various parameters so that large flakes incorporating with smaller ones is critically important to increase the ink conductivity.

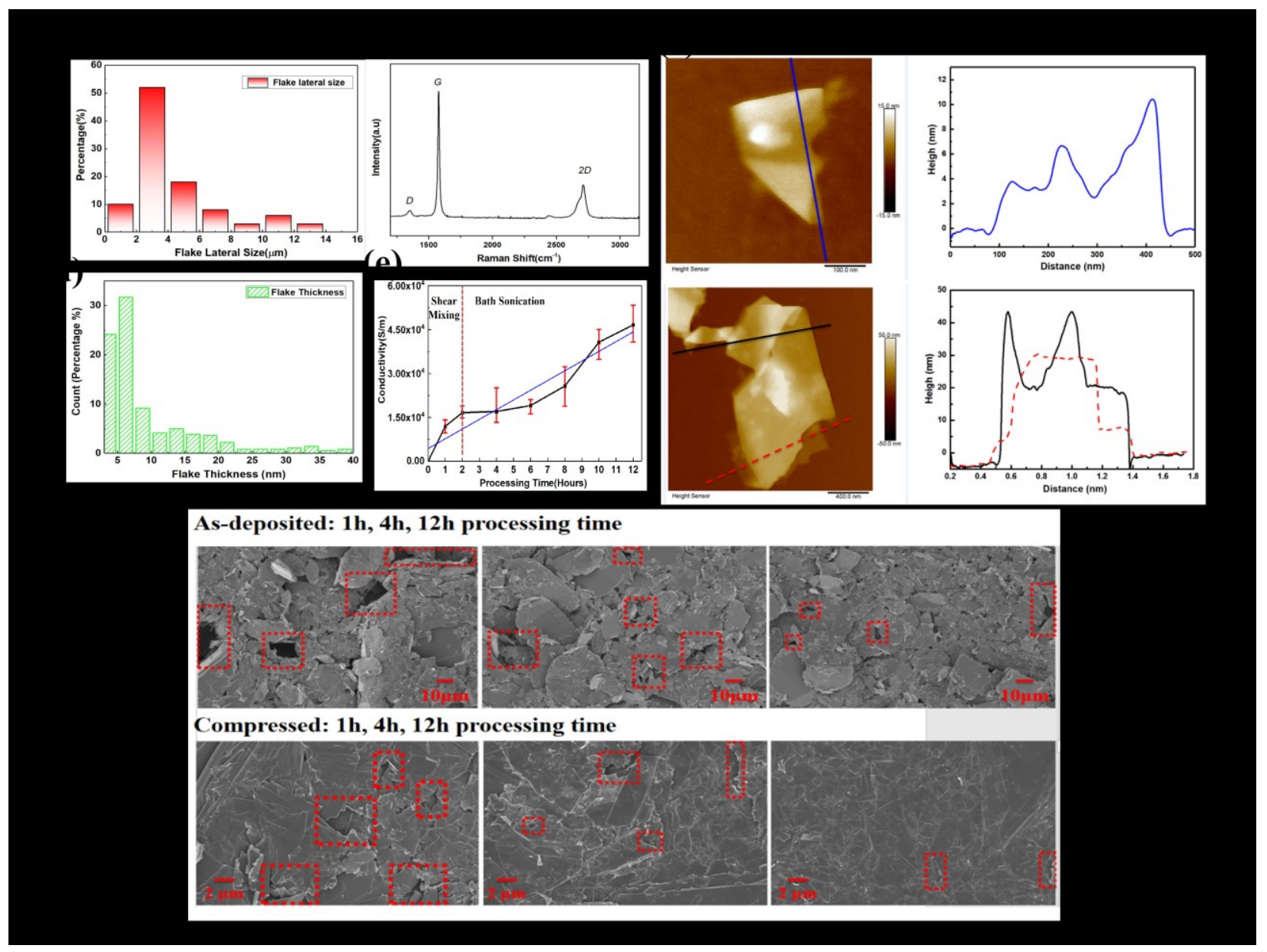

Figure 1. Characterization of GNP and printed samples. (a) Lateral size distribution of as prepared GNP counting 100 flakes (how long is the process time?); (b) Raman spectroscopy of GNP; (c) AFM topographic image of as-prepared individual GNP and surface scale curve; 
(d) Thickness distribution of prepared GNP counting 350 flakes; (e) Measured average GNP film conductivity values (measured five times per point) of conductivity as a function of sonication time. Red: Error bar; Blue: Linear regression of standard error estimate; (f) Comparison of surface topologies for as-deposited $(\times 2000$ magnification $)$ and compressed samples ( $\times 10000$ magnification) for $1 \mathrm{hr}, 4 \mathrm{hr}$ and $12 \mathrm{hr}$ processing time.

\subsection{Characterization of printed GNP on paper.}

The conductivity variations of the GNP laminate under different processing time in IPAwater mixed solvent is plotted in Figure 1e. As it can be seen, within the first $2 \mathrm{hr}$ processing time (shear mixing), the conductivity only has a subtle increase. It is only after $6 \mathrm{hr}$ processing time ( $2 \mathrm{hr}$ shear mixing $+4 \mathrm{hr}$ bath sonication) that the conductivity starts to increase notably and finally reaches $4.66 \times 10^{4} \mathrm{~S} / \mathrm{m}$ at around $12 \mathrm{hr}$ processing time $(2 \mathrm{hr}$ shear mixing $+10 \mathrm{hr}$ bath sonication), which is good enough for low-cost RF applications as reported previuosly ${ }^{8,10,13}$. The conductivity in Figure 1e was calculated based on measured sheet resistance and average thickness of the printed GNP laminates. The thickness of GNP laminates was measured based on SEM images (Supporting Information Figure S4). The average thickness was obtained by averaging the thickness measured at 5 different locations of the GNP laminate. The fitted linear regression line of standard error estimate is also shown in Figure 1e. The average error in percentage is calculated as $18 \%$ based on mean square error.

The GNP conductivity change with processing time can be explained intuitively by observing SEM surface topology as shown in Figure 1f. It can be seen that with $1 \mathrm{hr}$ 
processing time ( $1 \mathrm{hr}$ shear mixing), the graphite flakes have only sheared down to graphite particle-like structure, deep void alike holes are present due to ineffective inter-flake connections. As the processing time increases to $5 \mathrm{hr}$ and $12 \mathrm{hr}$, the voids become smaller and shallower, indicating better flake interconnecting and alignments. Higher magnification $(\times 10000)$ is used in order to better observe the topology change of the compressed samples. It can be seen that the samples with less processing time, even after compression, dents and cracks are still present because the flakes have collapsed into the voids during compression. The uncompressed samples for $1 \mathrm{hr}, 4 \mathrm{hr}, 12 \mathrm{hr}$ processing time can be seen from SEM $(\times 10,000$ magnification $)$ in Figure S3. These results demonstrate the significance of the flake size distributions. The un-exfoliated flakes are too thick and of large size, being difficult to stack up smoothly and jeopardizing the conductivity. The thinner and exfoliated flakes can stack well with a good size distribution where the bigger size flakes can act as the backbone and smaller flakes are utilized to fill to the voids. The thickness of screen printed samples after compression can be viewed from cross-sectional SEM provided in Figure S4. The average thickness is around $25 \mu \mathrm{m}$. The sheet resistance for the screen printed samples was measured to be $0.875 \Omega / \mathrm{sq}$. The conductivity is calculated to be $4.57 \times 10^{4} \mathrm{~S} / \mathrm{m}$, agrees well with the values shown in Figure 1e by dispersing GNP onto filtered paper. For UHF RFID applications operating at around $900 \mathrm{MHz}$, the skin depth is $78.3 \mu \mathrm{m}$ with conductivity around $4.6 \times 10^{4} \mathrm{~S} / \mathrm{m}$. The sheet resistance achieved here $(<1 \Omega / \mathrm{sq})$ is sufficient for $\mathrm{RF}$ applications with thickness only $32 \%$ of its skin depth ${ }^{4,38}$. Further improvement to reduce RF losses can be achieved by increasing the amount of ink deposition to increase the thickness but this will increase the cost in production. 
Concerning long time storage of the GNP conductive ink, the low-temperature treatment reported provides a possible way to avoid problems such as instability and aggregation for mixed solvent systems in dispersions ${ }^{21}$. The ink prepared for this work has been stored for 3 months in laboratory fridge operating at $-18^{\circ} \mathrm{C}$ and the printed samples show no obvious performance degradation.

The concentrated GNP conductive ink is suitable for screen printing (the viscosity data can be found in Supporting Information Figure S5). The ink is highly shear thinning, with a power law exponent close to -1 . The highly shear thinning property of the ink helps to quickly adjust the viscosity for printing even at a lower printing speed for ink deposition.

For the printed samples using GNP ink, performance degradation is not observed over 6 months. Different from inks made of graphene oxide (GO) and reduced graphene oxide (rGO), where the functional groups can have effect on water absorbance, GNP printed patterns on papers reported in this work are very stable. Tape peel tests have also been carried out on printed samples using wafer tape BT-50E-FR, wafer tape BT-150E-KL and scotch packing tape to test the film adhesion on paper. As shown in Supporting Information Figure S6, the samples can endure 10 times peel with wafer tapes and remain usable sheet resistance $(3 \mathrm{ohm} / \mathrm{sq})$ but sheet resistance increase several orders $(600 \mathrm{ohms} / \mathrm{sq})$ after peeling 8 times with scotch packing tape. This reveals that the printed patterns in this work has the ability to endure small scratches or rubs. Characterization of line and gap resolution with different layers are also shown in Supporting Information Figure S7 and Figure S8, demonstrating that sufficient printing resolution has been achieved for RF applications. The thickness of the printed GNP is mainly controlled by the thickness or layers of capillary film. As shown in Supporting Information Figure S9, the GNP laminate thickness increases 
monotonically with more layers of the capillary film. The GNP laminate thickness also depends on other factors such as printing speed, snap and lift off distance, screen thickness, design patterns, squeegee type and pressure, etc.

Direct exfoliation in processable low-boiling point solution without re-dispersion for solvent exchange is desirable, i.e., one-pot ink synthesis. The mixed solvent of IPA-water also provides a way of easy removal after pattern printing by mild heating for drying the printed samples ${ }^{39}$, which further reduces the manufacturing costs.

\subsection{GNP conductive ink printed UHF RFID tag and reader antennas}

The prepared conductive ink has been used to print RFID tag and reader antennas with satisfactory performance. GNP conductive ink screen printed RFID tag antennas with mid-

long range are designed with T-matching and nested slot techniques ${ }^{40-42}$. For best matching between the tag antenna and the bonded RFID chip, the impedances of the antenna and the chip should be conjugately matched at the operating frequency. The matching factor can be expressed as:

$$
\tau=\frac{4 R_{c} R_{a}}{\left|Z_{c}+Z_{a}\right|^{2}}
$$

where $Z_{c}=R_{c}+j X_{c}$ is the impedance of the RFID chip and $Z_{a}=R_{a}+j X_{a}$ is the tag antenna impedance. Full-wave electromagnetic simulations using CST 2017 for both mid and long range RFID tag antennas are performed as shown in Figure 2a and Figure 2b, respectively. As it can be seen, the tag antennas are conjugately matched to the RFID chip 
impedance at around $900 \mathrm{MHz}$. For commercial RFID tag applications, the read range at which the RFID reader can detect the backscattered signal from the tag is the most important tag performance indicator ${ }^{10,13}$. The reading range $r$ of a RFID system can be calculated by ${ }^{43}$,

$$
r=\frac{\lambda}{4 \pi} \sqrt{\frac{P_{t} G_{t} G_{r} \tau}{P_{t h}}}
$$

where $\lambda$ is the wavelength, $P_{t}$ is the transmitted power from reader antenna, $G_{t}$ is the gain of reader antenna, $G_{r}$ is the gain of tag antenna, $P_{t h}$ is the minimum threshold of the power needed to active the RFID chip. The geometry dimensions of the prototype antennas are shown in Figure 2c and Figure 2d, respectively. The results of Eq (2) were calculated from the full wave simulated real and imaginary part of the tag antenna impedance. The measured read ranges of the prototypes were measured using commercially available Voyantic RFID Tagformance measurement system with EIRP $=4 \mathrm{~W}$. The simulated and measured results are displayed in Figure 2e. Before measurement, the system was calibrated and the effects of environments, cable and free-space loss were eliminated. The system swept linearly from $860 \mathrm{MHz}$ to $960 \mathrm{GHz}$ with $1 \mathrm{MHz}$ step for read range measurement. The mid-range printed graphene RFID prototype, can achieve $5 \mathrm{~m}$ read range in the whole RFID band and reaches around $6 \mathrm{~m}$ at $920 \mathrm{MHz}$, whilst the long range one can achieve up to $9 \mathrm{~m}$ read range. The measurement results demonstrate that the low cost, green production of GNP conductive ink printed RFID tag antennas can provide satisfactory performance in mid to long range applications. The results are comparable to those published works ${ }^{10,13}$. 


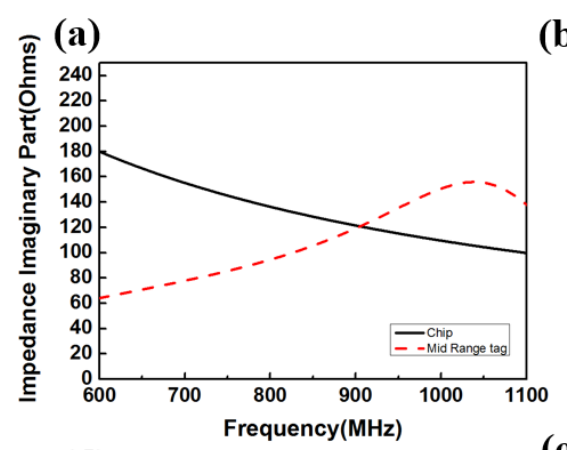

(b)

(d)

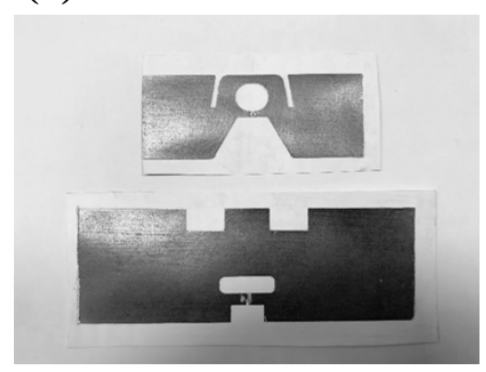

e)

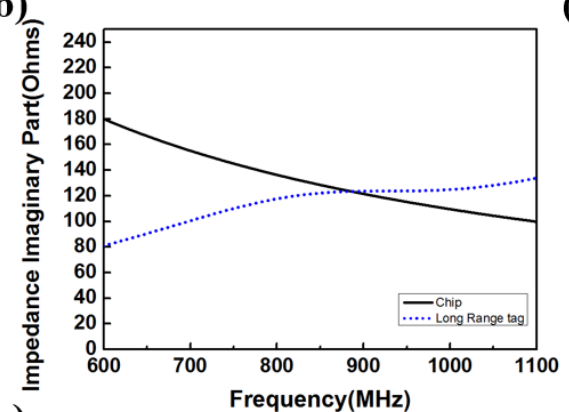

(c)
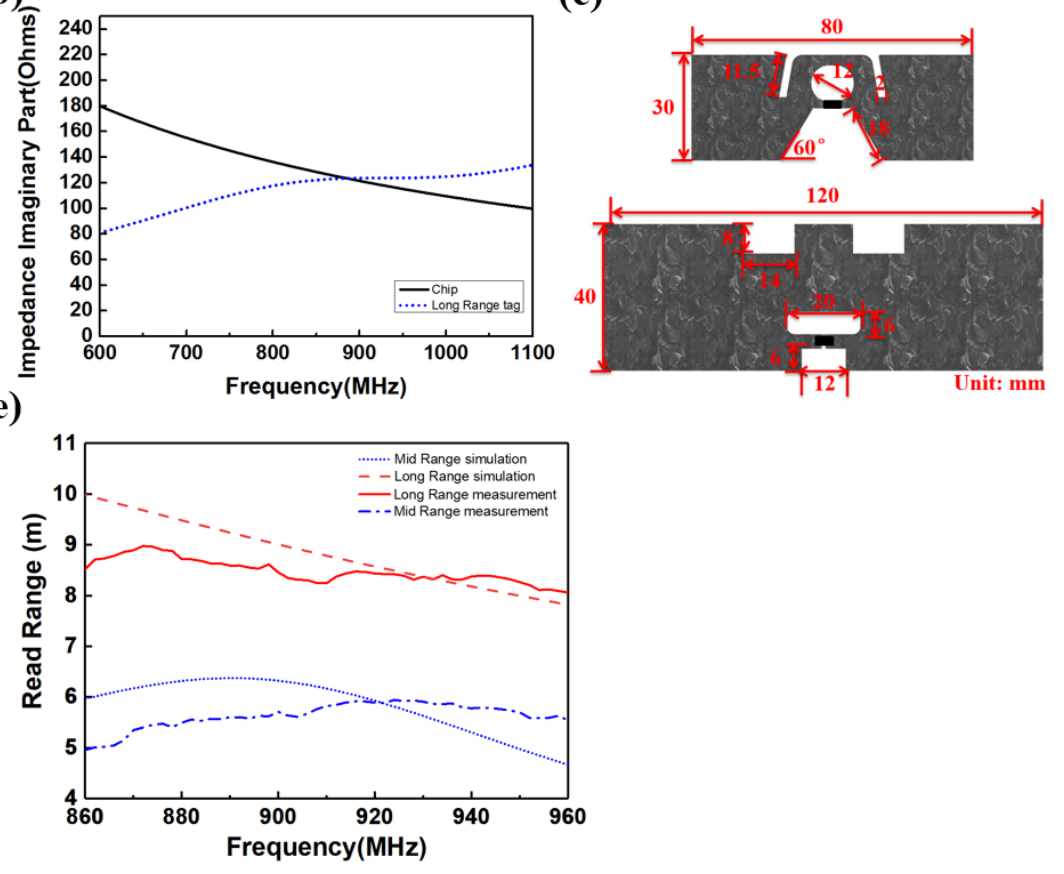

Figure 2. GNP conductive ink screen printed RFID tags. (a) Simulated impedance matching for mid-range RFID tag; (b)Simulated impedance matching for long-range RFID tag; (c) Geometry dimensions and structures for GNP ink printed RFID tags; (d) GNP conductive ink screen printed RFID mid and long range tag prototypes; (e) Measured read ranges for the printed RFID mid and long range tag prototypes. 

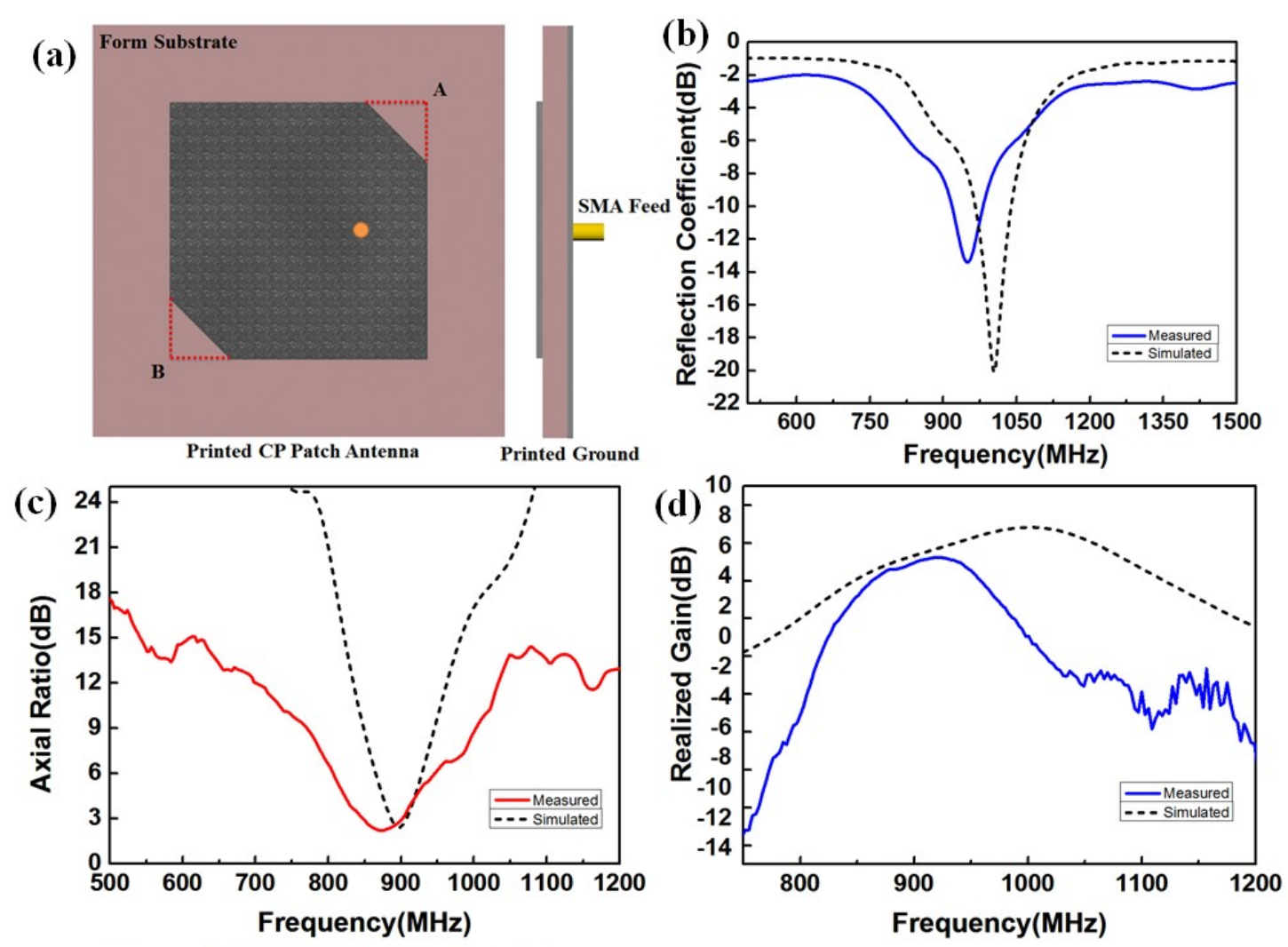

(e)

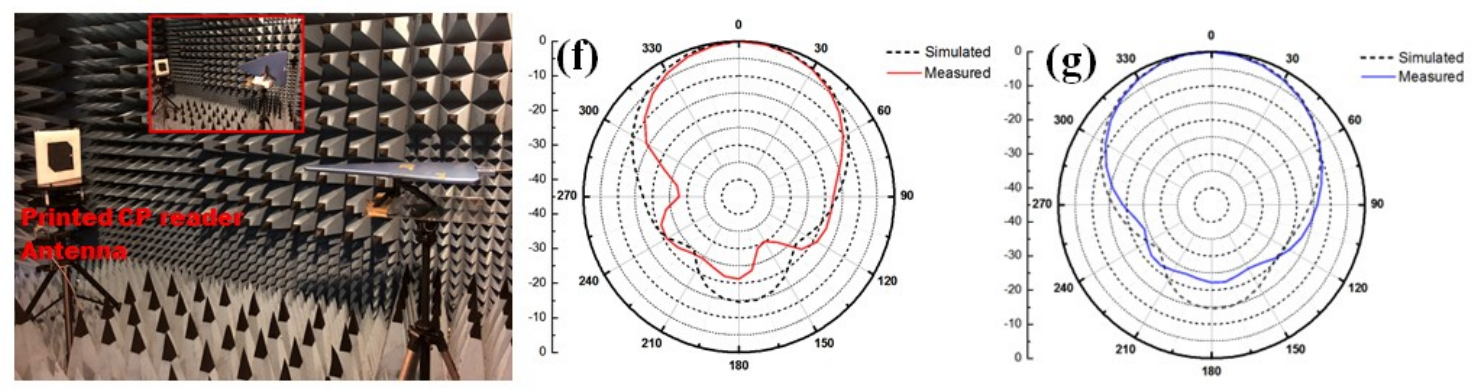

Figure 3. GNP conductive ink screen printed RFID reader antenna. (a) Printed graphene CP patch antenna structure; (b) Measured and simulated reflection coefficient of the GNP ink printed CP antenna; (c) Measured Axial Ratio of GNP ink printed CP antenna; (d)Measured realized gain of printed $\mathrm{CP}$ antenna; (e) Measurements of printed $\mathrm{CP}$ antenna prototype in microwave anechoic chamber; (f) Measured spinning linear radiation patterns at $900 \mathrm{MHz}$ in the $\mathrm{x}-\mathrm{z} ;(\mathrm{g})$ Measured spinning linear radiation patterns at $900 \mathrm{MHz}$ in the $\mathrm{y}-\mathrm{z}$ planes. 
A reader antenna is essential in RFID system. RFID tag antennas receive the RF signal transmitted by the reader antenna and tag antennas are typically linearly polarized. A linearly polarized antenna only operates well and receives maximum power when the RFID tag antenna's polarization matches the reader antenna's polarization. Hence circularly polarized (CP) reader antennas are commonly used so that polarization mismatch can be eliminated. For a $\mathrm{CP}$ antenna, the axial ratio (AR) is crucially important. It is defined as the ratio between the minor and major axis of the polarization of the antenna. The ideal value of the axial ratio is $0 \mathrm{~dB}$. Practically, AR below $3 \mathrm{~dB}$ is acceptable ${ }^{44,45}$. In this work, a GNP printed CP reader antenna has been designed, printed and characterized for the first time.

The layout and characterization of the $\mathrm{CP}$ reader antenna is shown in Figure 3. The circular polarization is realized by exciting two orthogonal degenerate resonant modes in phase quadrature by truncating the two opposite corners of the square patch $^{46}$. The sides of the square patch (i.e., $L=W=140 \mathrm{~mm}$ ), and of $\approx 0.42$ times of free-space wavelength at $900 \mathrm{MHz}$. The corner of the square patch is truncated inwards by $22 \mathrm{~mm}$ at points $\mathrm{A}$ and B, left hand circular polarization is obtained at broadside. The antenna is fed by a $50 \Omega$ SMA panel mount connector (RS Pro, 921-0219), shown in Figure 3a. The connector is connected to the radiating patch and the ground plane by conductive epoxy (Circuitworks, CW2400). The substrate is industrial grade rigid foam (ROHACELL, 71IG-F) with dielectric constant $\sim \varepsilon=1.08$. The foam is of the same size as the ground plane $\left(200 \times 200 \mathrm{~mm}^{2}\right)$, with a height of $15 \mathrm{~mm}$. The antenna layout as well as the ground plane were GNP ink screen printed on normal A4 printing papers and stacked on both sides of the foam. The CP reader antenna was measured in the anechoic chamber. The measured reflection coefficient $\left(\mathrm{S}_{11}\right)$ (using Vector Network Analyzer, Keysight E5071B), shown in Figure 3b, reveals that the 
antenna resonates at $950 \mathrm{MHz}$, with a $-10 \mathrm{~dB}$ bandwidth of $66 \mathrm{MHz}(917-983 \mathrm{MHz}$, which implies that more than $90 \%$ power is radiated from the antenna in this band). Figure $3 \mathrm{c}$ shows the measured and simulated axial ratio in the broadside direction of the $\mathrm{CP}$ reader antenna. The minimum axial ratio is observed at $875 \mathrm{MHz}$ with $2.21 \mathrm{~dB}$. The measured 3-dB axial-ratio bandwidth shows $57 \mathrm{MHz}(845-902 \mathrm{MHz})$ or about $10.4 \%$ with respect to 875 $\mathrm{MHz}$ at which the minimum axial ratio is observed. The measured axial ratio is a slight shift from the simulated. This is most likely due to the parasitic inductance introduced by the feeding probe ${ }^{47}$. Nevertheless, the performance of the CP reader antenna is still satisfactory and circular polarization operational frequency still lies in the RFID UHF band.

There is a mismatch between the $3 d B$ AR simulated and measured bands, which can be overcome by further optimization of the $\mathrm{CP}$ reader antenna design. In the simulation, the material is modelled as ohmic sheet of $1 \Omega /$ sq. In the actual case, variations in sheet resistance will be caused by the exposed screen and also the ink deposition. In addition, the SMA connector is connected to the antenna using conductive epoxy, whereas the connection was set to be perfect in the simulation. The performance of the $\mathrm{CP}$ reader antenna is further studied by looking into the gain measurement and radiation patterns as shown in Figure $3 \mathrm{~d}-$ 3g. The gain measurement was carried out by transfer gain method with a reference linear polarized antenna (HyperLOG 6080, $680 \mathrm{MHz}-8 \mathrm{GHz}$ ). The gain of the reference antenna is known so the gain of the reader antenna can be obtained by Eq (4) and further calculated with a correction factor to account for the nature of the circular polarization as shown in Eq (7) to compensate for the finite AR because practical antennas generate cross-polar power, which results in an elliptically polarized field pattern ${ }^{48}$.

$$
\operatorname{Gain}_{\text {Receiver }}(d B)=S_{21}(d B)-P L(d B)-\operatorname{Gain}_{\text {Transmitter }}(d B)
$$




$$
\begin{aligned}
& P L(d B)=20 \log \left(\frac{\lambda}{4 \pi r}\right) \\
& \operatorname{Gain}_{\text {correction }}(d B)=20 \log \left[0.5\left(1+10^{-\frac{A R}{20}}\right)\right](d B) \\
& \operatorname{Gain}_{A U T}(d B i c)=\operatorname{Gain}(d B)+3 d B+\operatorname{Gain}_{\text {correction }}(d B)
\end{aligned}
$$

From Figure $3 \mathrm{~d}$ it can be observed that the peak gain of $2.63 \mathrm{dBic}$ occurs at $902 \mathrm{MHz}$, and above $0 \mathrm{dBic}$ between $842 \mathrm{MHz}$ and $962 \mathrm{MHz}$, covering most RFID bands, especially 865-868 and 902-928 MHz. The effective radiation is verified by far field radiation pattern measurement performed in anechoic chamber with a rotary table tripod (Diamond Engineering, D6000) and measured with Diamond Engineering antenna measurement system. The radiation patterns on $\mathrm{x}-\mathrm{y}$ plane and $\mathrm{y}-\mathrm{z}$ plane have been obtained. The measurements are performed at a 10 degrees step and normalized with maximum received power. The zero degree corresponds to the direction normal to the CP reader antenna. The results agree well with the simulated radiation patterns in the $x-y$ plane and $y-z$ plane, the minor mismatch and slight asymmetry in the back lobe are considered due to the presence of the SMA cables in the back. The radiation patterns are of typical shape of a patch antenna with the main beam radiating outwards from the radiation patch and a nominal back lobe from the antenna ground. Comparisons between this work against commercial UHF RFID antennas and research works are given in Supporting Information Table S1 (Tags) and Table S2 (Reader antenna). Advantages and disadvantages of metal and GNP printed tag antennas are provided in Supporting Information Table S3. In short, the GNP printed RFID antennas provide a low cost, environmentally friendly alternative fabrication method and material to the traditional metal RFID antennas with satisfactory performance. 
3.4. Machine learning enabled GNP conductive ink printed RFID for sensing unidentified

\section{liquids}

UHF RFID tag antenna inspired by square split ring resonator structure from previous work is used as the sensing structure ${ }^{49}$. Resonator structures with slots are very appropriate for fluidics-based sensing as loading the slot with dielectric materials with different permittivity $\varepsilon$ will change its capacitance ${ }^{50}$. The electrochemical and electro physical properties of liquids (e.g., molecules and ions in the material) affect material dielectric permittivity hence the ability to store surrounding electromagnetic field is different for different liquids ${ }^{51}$. When a centrifuge tube filled with a liquid is placed in the slots of the RFID resonator tag, as shown in Figure 4a, the electromagnetic field of the RFID tag is affected due to the coupling fields and proximity effects. This can be clearly observed in the electric field strength shown in Figure 4b. The electric field is very strong in the slots of the resonator ring without tube loading (Top of Figure 4b), but when a tube filled with dielectric liquid, such as solvent, is placed on the top and in the middle of the ring, as shown in Figure 4a, the electric field strength becomes much weaker due to the dielectric loss and mismatch. The RF receiver at the RFID reader can retrieve the backscattered signal waveform hence the backscattered power and phase can be analyzed. Same as electric field strength, the backscattered power will suffer from dielectric loss and electromagnetic absorption by the solvents $^{52,53}$. It has been reported that the backscattered phase change from the RFID tag can be used to analyze humidity change when layered with humidity sensitive material $\mathrm{GO}^{31}$ and food contamination when attached to packaging ${ }^{51}$. Most commercial RFID readers can perform this because RFID readers perform fully coherent detection and are capable of recovering the baseband phase of the coherently demodulated backscattered signal ${ }^{54,55}$. 
Hence, RF measurement data such as electric field strength, backscattered power and backscattered phase can be extracted for sensing purposes.

(a)

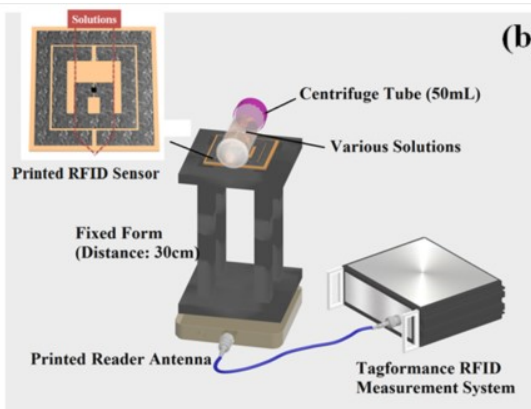

(c)

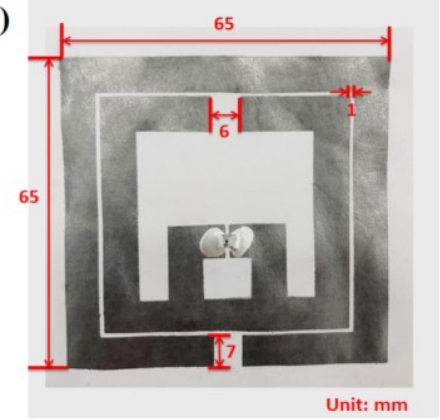

(b)
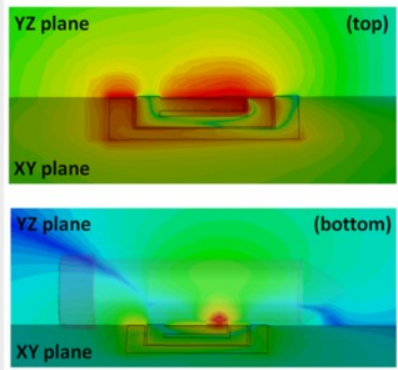

(d)

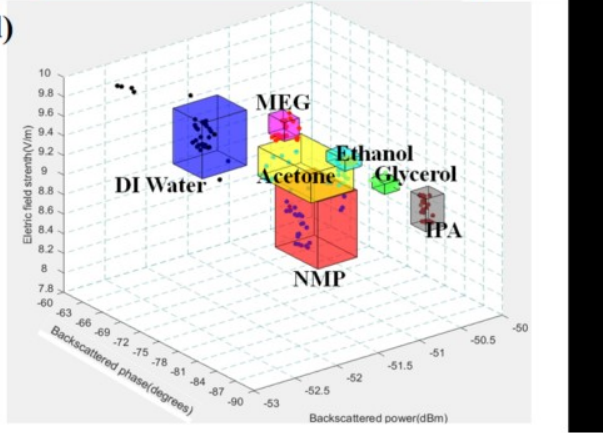

(e)

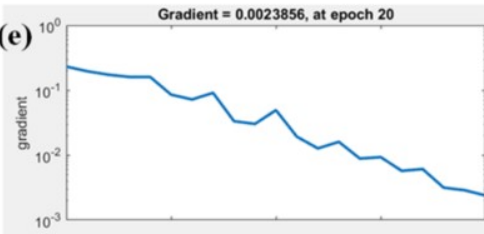

(f)
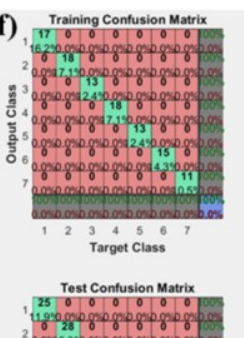

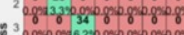

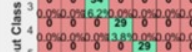

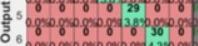

2
70

$\begin{array}{llll}3 & 4 & 5 & 6 \\ \text { Target Class } & 7\end{array}$

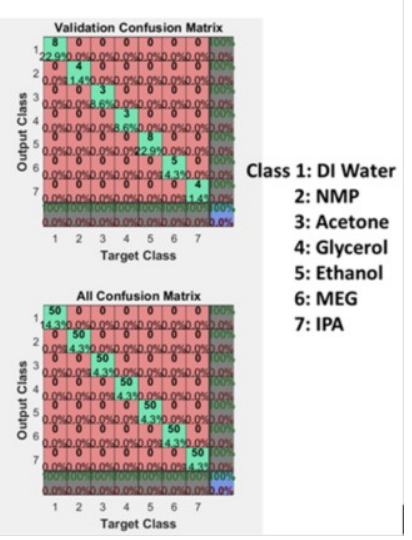

Figure 4. Machine learning enabled battery-free wireless RFID Sensing system for

liquid classification application. (a) Printed sensor tag and reader antenna RFID sensing system setup; (b) Electric field of the printed sensor tag, top: no liquid tube, bottom: liquid tube loaded; (c) GNP ink Printed sensor tag antenna prototype and dimensions; (d)3D illustration of 3-dimensional training data range (described by box with different colors) and test data (filled color dots); (e) Training record using scaled conjugate gradient 
backpropagation, top: Gradient approaching of 20 epochs, bottom: validation check of 20 epochs; (f) Confusion matrixes for self-validation of training data and evaluation of test data.

However, the variation of backscattered phase for different loaded liquids (or indeed other parameters) is normally very weak and measurements with one parameter for different liquids can fall into the same small range ${ }^{51}$. The classification task becomes all the more difficult for identifying multiple objects based on just one parameter hence the necessity to introduce machine learning with the ability to train with more parameters and accurately identify the patterns even with small variations.

Machine learning has emerged as the method of choice for developing applications that provide systems the ability to access data and automatically learn and improve from training experience without being statistics programmed ${ }^{56}$. Machine leaning has different frameworks based on different algorithms such as Feed-forward Neural Networks (FNN), Support Vector Machines (SVM) and Adaptive Boosting (Adaboost) ${ }^{57}$. Back propagation (BP) pattern recognition neural networks is a type of FNN that can be trained to classify input patterns according to target classes by back-propagating errors from one network layer to another so to adjust and amend the network and finally approaches minimum error and the correct output $^{58}$. A neural network can consist of a series of layers to solve the input-output mapping problem. Each subsequent layer has a connection from the previous layer. The data are fed through the network and the network's output is produced as the predicted pattern of the input data ${ }^{59}$.

The ability to identify an unlabeled colorless transparent liquid is of essential usefulness to chemical and medical disposal to prevent hazards. In our experiment, $50 \mathrm{~mL}$ centrifuge 
tube (VWR) are filled with 7 different colorless transparent solvents including Deionized (DI) water, N-Methyl-2-pyrrolidone (NMP), Acetone, Glycerol, Ethanol, Ethylene glycol (MEG), Isopropyl alcohol (IPA). GNP ink printed RFID tag antenna, shown in Figure 4c, was used and loaded with the centrifuge tubes. For each solvent, 50 data sets were measured with Voyantic RFID measurement system and recorded including electric field strength, backscattered power and backscattered phase. Inputs are 3-element vectors corresponding to the 3 input data values (electric field strength, backscattered power and backscattered phase). The number of output neurons is set to 7 , which is equal to the number of elements in the target vector (the number of categories), corresponding to 7 different solvents (Deionized (DI) water, N-Methyl-2-pyrrolidone (NMP), Acetone, Glycerol, Ethanol, Ethylene glycol (MEG), Isopropyl alcohol (IPA)).

These data were fed into the neural network for machine learning to determine the content filled in the tube. 350 input data sets in total were randomized, as shown in the 3D mapping grid in Figure 4d, the squares represent the range of the training data, and the dots represent the test data. It can be seen that some test data is outside of the range, and some data is mixing with each other, e.g. MEG, Ethanol and Acetone. The three dimension data can increase the cross-checking accuracy however the scattered non-linear 3D data would be too difficult for classification task with a weak classifier operating on basic inequality operators ${ }^{60}$. Hence, a two-layer BP feedforward network, with a sigmoid transfer function in the hidden layer, and a softmax transfer function in the output layer, trained with scaled conjugate gradient backpropagation were used to process the data. To achieve better recognition and classification, the hidden layer has been set to 20 .

Among the 350 input data sets, 105 data sets (30\%) were used for training and 35 data sets $(10 \%)$ were for validation of the training, and the rest 210 data sets $(60 \%)$ were to blind test the performance. It can be seen from the training state shown in Figure 4e, according to scaled conjugate gradient descent, the gradient performance reduces from 0.328 to 0.00238 
after 20 epochs. The validation checks only fail 3 times in all 20 epochs, and reach to zero at the end. From the confusion matrix depicted in Figure 4f, the accuracy can reach $100 \%$ at the end for all predictions. This means after training, when a centrifuge tube filled with an unknown solvent is placed on the RFID tag, it can be distinguished clearly and labelled. Knearest neighbor (KNN) method and Support Vector Machines (SVM) were also implemented for comparisons. The accuracy for $\mathrm{KNN}$ ranges from $90 \%$ to $96 \%$ using Minkowski metric, depending on the neighbor size (2 to 10). The accuracy for SVM using One-against-all (OAA) strategy with Polynomial kernel ranges from $88 \%$ to $97 \%$, depending largely on weight penalty ( 0.1 to 20$)$. The trained BP neural network can reach $100 \%$ accuracy with 20 hidden layers. It is preferable that higher accuracy should be used in wireless sensing applications. This wireless battery-free RFID sensing for liquid classification has huge potential in chemical detection and medical healthcare, where the heavy use of solvents and liquid chemicals takes place. The mechanism can be even popularized to every aspect of life that has potential requirement for wireless sensing, such as orientation and localization, structural health and environmental monitoring. Combining BP neural network with RFID resonator tag, printed by scalable, cheap and green processible GNP conductive ink, affordable and omnipresent RFID wireless sensing applications could be in sight for everyday use.

\section{Conclusions}

In conclusion, this work demonstrates that screen printing GNP conductive ink prepared by low-boiling point IPA-water mixture without solvent exchange can have high conductivity and be used to print low-cost and flexible RFID tag and reader antennas for 
classification sensing applications. Without the need of using toxic or high boiling point solvents, the process is green, easy and low cost. The GNP conductive ink printed RFID tag and reader antennas can provide the required functionalities. The mid and long range RFID tag can provide $6 \mathrm{~m}$ and $9 \mathrm{~m}$ read range, respectively. CP reader antenna has 3-dB axial-ratio bandwidth of $57 \mathrm{MHz}(845-902 \mathrm{MHz}$ ), and peak gain of $2.63 \mathrm{dBic}$. Furthermore, low cost RFID solvent classification sensing system has been proposed and verified experimentally for labelling and identifying transparent and colorless liquids, showing $100 \%$ accuracy with the aid of screen printed RFID resonator tag and neural network machine learning. This low cost classification sensing system can be of vast use to medical and chemical related works and have potential to be used in IoT wireless sensing for a great diversity of applications such as structural health and food safety monitoring. Overall, this work demonstrates that the GNP conductive ink prepared by low cost, low boiling point, green and low toxicity mixed solvent of IPA-water without any solvent exchange can provide satisfactory performance in screen printing RFID antennas for low cost classification sensing system and has the potential to replace carbon based conductive ink prepared using expensive, toxic, and high boiling point solvents.

\section{AUTHOR INFORMATION}

\section{Corresponding Author}

* Zhirun Hu

Email: z.hu@manchester.ac.uk 


\section{Author Contributions}

T.L. and Z.H. designed and prepared the experiments, measured and analyzed the experimental data and drafted the manuscript. K.P. carried out the SEM, AFM and Raman measurements and participated in discussions and drafted the manuscript. D.Z., S.A. and J.L. drafted the manuscript. K.S.N. coordinated the project as well as drafted the manuscript.

\section{Notes}

The authors declare no financial interests.

\section{Acknowledgements}

The project is supported by UK Engineering and Physical Research Council

(EPN010345), the Royal Society, EU Graphene Flagship Program, European Research

Council Synergy Grant Hetero2D, US Army Research Office (W911NF-16-1-0279) and

NMS.

\section{Supporting Information}

Figures

Figure S1 - Raman spectroscopy measured on smaller flake. A Higher D/G ratio indicating structural defects and edge disturbance. 
Figure S2 - AFM image for thickness distribution analysis.

Figure S3 - Fig. S3 - SEM image of uncompressed samples for (a) 1h, (b) $4 \mathrm{~h}$, (c) $12 \mathrm{~h}$ processing time $(\times 10,000$ magnification) and (d) $1 \mathrm{~h}$, (e) $4 \mathrm{~h}$, (f) $12 \mathrm{~h}$ processing time $(\times 20,000$ magnification).

Figure S4 - Cross sectional SEM image of compressed screen printed samples.

Figure S5 - Viscosity measurement for concentrated ink $(50 \mathrm{mg} / \mathrm{mL})$. The viscosity is sufficient to be used for screen printing ( $\mu \sim 0.5-5$ Pas).

Figure S6 - Tape peel test with wafer tape BT-50E-FR, wafer tape BT-150E-KL and scotch packing tape.

Figure S7 - Printing resolution of different screen coating layers.

Figure S8 - Screen coating thickness (2 layer, 3 layer and 4 layer coating) and printed GNP film thickness ( $\times 500$ magnification).

Tables

Table S1 - Comparisons against commercial UHF RFID tag antennas and research works

Table S2 - Comparisons against commercial UHF RFID reader antennas and research works

Table S3 - Advantages and disadvantages of metal and GNP printed antennas

Experimental Notes

1. Tape peel test

2. Printing resolutions

3. Antennas and electric field simulations

4. Machine Learning Implementation

5. RFID tag comparisons 


\section{References}

(1) Saidina, D. S.; Eawwiboonthanakit, N.; Mariatti, M.; Fontana, S.; Rold, C. H. Recent Development of Graphene-Based Ink and Other Conductive Material-Based Inks for Flexible Electronics. 48. https://doi.org/10.1007/s11664-019-07183-w.

(2) Stafford, J.; Patapas, A.; Uzo, N.; Matar, O. K.; Petit, C. Towards Scale-up of Graphene Production via Nonoxidizing Liquid Exfoliation Methods. AIChE J. 2018, 64 (9), 3246-3276.

https://doi.org/10.1002/aic.16174.

(3) Ejigu, A.; Miller, B.; Kinloch, I. A.; Dryfe, R. A. W. Optimisation of Electrolytic Solvents for Simultaneous Electrochemical Exfoliation and Functionalisation of Graphene with Metal Nanostructures. Carbon N. Y. 2018, 128, 257-266. https://doi.org/10.1016/J.CARBON.2017.11.081.

(4) Ng, L. W. T.; Hu, G.; Howe, R. C. T.; Zhu, X.; Yang, Z.; Jones, C. G.; Hasan, T. Printing of Graphene and Related 2D Materials: Technology, Formulation and Applications; Springer International Publishing: Cham, 2019. https://doi.org/10.1007/978-3-319-91572-2.

(5) Ortego, I.; Sanchez, N.; Garcia, J.; Casado, F.; Valderas, D.; Sancho, J. I. Inkjet Printed Planar Coil Antenna Analysis for NFC Technology Applications. Int. J. Antennas Propag. 2012, 2012, 1-6. https://doi.org/10.1155/2012/486565.

(6) Nishi, Y.; Doering, R.; Doering, R. Handbook of Semiconductor Manufacturing Technology, Second Edition; Doering, R., Nishi, Y., Eds.; CRC Press, 2007. https://doi.org/10.1201/9781420017663.

(7) Khan, S.; Lorenzelli, L.; Dahiya, R. S. Technologies for Printing Sensors and Electronics Over Large Flexible Substrates: A Review. IEEE Sens. J. 2015, 15 (6), 3164-3185.

https://doi.org/10.1109/JSEN.2014.2375203.

(8) Huang, X.; Leng, T.; Zhang, X.; Chen, J. C.; Chang, K. H.; Geim, A. K.; Novoselov, K. S.; Hu, Z. BinderFree Highly Conductive Graphene Laminate for Low Cost Printed Radio Frequency Applications. Appl. Phys. Lett. 2015, 106 (20), 203105. https://doi.org/10.1063/1.4919935.

(9) Huang, X.; Leng, T.; Zhu, M.; Zhang, X.; Chen, J.; Chang, K.; Aqeeli, M.; Geim, A. K.; Novoselov, K. S.; $\mathrm{Hu}, \mathrm{Z}$. Highly Flexible and Conductive Printed Graphene for Wireless Wearable Communications Applications. Sci. Rep. 2016, 5 (1), 18298. https://doi.org/10.1038/srep18298.

(10) Leng, T.; Huang, X.; Chang, K.; Chen, J.; Abdalla, M. A.; Hu, Z. Graphene Nanoflakes Printed Flexible Meandered-Line Dipole Antenna on Paper Substrate for Low-Cost RFID and Sensing Applications. IEEE Antennas Wirel. Propag. Lett. 2016, 15, 1565-1568. https://doi.org/10.1109/LAWP.2016.2518746. 
(11) Huang, X.; Leng, T.; Chang, K. H.; Chen, J. C.; Novoselov, K. S.; Hu, Z. Graphene Radio Frequency and Microwave Passive Components for Low Cost Wearable Electronics. 2D Mater. 2016, 3 (2), 025021. https://doi.org/10.1088/2053-1583/3/2/025021.

(12) Merilampi, S.; Laine-Ma, T.; Ruuskanen, P. The Characterization of Electrically Conductive Silver Ink Patterns on Flexible Substrates. Microelectron. Reliab. 2009, 49 (7), 782-790. https://doi.org/10.1016/J.MICROREL.2009.04.004.

(13) Pan, K.; Fan, Y.; Leng, T.; Li, J.; Xin, Z.; Zhang, J.; Hao, L.; Gallop, J.; Novoselov, K. S.; Hu, Z. Sustainable Production of Highly Conductive Multilayer Graphene Ink for Wireless Connectivity and IoT Applications. Nat. Commun. 2018, 9 (1), 5197. https://doi.org/10.1038/s41467-018-07632-w.

(14) Nguyen, N.; Zhang, S.; Oluwalowo, A.; Park, J. G.; Yao, K.; Liang, R. High-Performance and Lightweight Thermal Management Devices by 3D Printing and Assembly of Continuous Carbon Nanotube Sheets. $A C S$ Appl. Mater. Interfaces 2018, 10 (32), 27171-27177. https://doi.org/10.1021/acsami.8b07556.

(15) Zhang, S.; Nguyen, N.; Leonhardt, B.; Jolowsky, C.; Hao, A.; Park, J. G.; Liang, R. Carbon-NanotubeBased Electrical Conductors: Fabrication, Optimization, and Applications. Adv. Electron. Mater. 2019, 5 (6), 1-36. https://doi.org/10.1002/aelm.201800811.

(16) Hernandez, Y.; Nicolosi, V.; Lotya, M.; Blighe, F. M.; Sun, Z.; De, S.; McGovern, I. T.; Holland, B.; Byrne, M.; Gun'Ko, Y. K.; et al. High-Yield Production of Graphene by Liquid-Phase Exfoliation of Graphite. Nat. Nanotechnol. 2008, 3 (9), 563-568. https://doi.org/10.1038/nnano.2008.215.

(17) O’Neill, A.; Khan, U.; Nirmalraj, P. N.; Boland, J.; Coleman, J. N. Graphene Dispersion and Exfoliation in Low Boiling Point Solvents. J. Phys. Chem. C 2011, 115 (13), 5422-5428. https://doi.org/10.1021/jp110942e.

(18) Salavagione, H. J.; Sherwood, J.; De bruyn, M.; Budarin, V. L.; Ellis, G. J.; Clark, J. H.; Shuttleworth, P. S. Identification of High Performance Solvents for the Sustainable Processing of Graphene. Green Chem. 2017, 19 (11), 2550-2560. https://doi.org/10.1039/C7GC00112F.

(19) Yi, M.; Shen, Z.; Ma, S.; Zhang, X. A Mixed-Solvent Strategy for Facile and Green Preparation of Graphene by Liquid-Phase Exfoliation of Graphite. J. Nanoparticle Res. 2012, 14 (8), 1003. https://doi.org/10.1007/s11051-012-1003-5.

(20) Liu, L.; Shen, Z.; Yi, M.; Zhang, X.; Ma, S. A Green, Rapid and Size-Controlled Production of HighQuality Graphene Sheets by Hydrodynamic Forces †. 2014. https://doi.org/10.1039/c4ra05635c.

(21) Liu, L.; Shen, Z.; Zhang, X.; Ma, S. Low-Temperature Treatment for Preservation and Separation of Graphene Dispersions. J. Mater. Sci. 2018, 53 (19), 13875-13885. https://doi.org/10.1007/s10853-018$2572-1$. 
(22) Deegan, R. D.; Bakajin, O.; Dupont, T. F.; Huber, G.; Nagel, S. R.; Witten, T. A. Capillary Flow as the Cause of Ring Stains from Dried Liquid Drops. Nature 1997, 389 (6653), 827-829.

https://doi.org/10.1038/39827.

(23) Dong, L.; Chen, Z.; Zhao, X.; Ma, J.; Lin, S.; Li, M.; Bao, Y.; Chu, L.; Leng, K.; Lu, H.; et al. A NonDispersion Strategy for Large-Scale Production of Ultra-High Concentration Graphene Slurries in Water. Nat. Commun. 2018, 9 (1), 76. https://doi.org/10.1038/s41467-017-02580-3.

(24) Hyun, W. J.; Secor, E. B.; Hersam, M. C.; Frisbie, C. D.; Francis, L. F. High-Resolution Patterning of Graphene by Screen Printing with a Silicon Stencil for Highly Flexible Printed Electronics. Adv. Mater. 2015, 27 (1), 109-115. https://doi.org/10.1002/adma.201404133.

(25) Karagiannidis, P. G.; Hodge, S. A.; Lombardi, L.; Tomarchio, F.; Decorde, N.; Milana, S.; Goykhman, I.; Su, Y.; Mesite, S. V.; Johnstone, D. N.; et al. Microfluidization of Graphite and Formulation of GrapheneBased Conductive Inks. ACS Nano 2017, 11 (3), 2742-2755. https://doi.org/10.1021/acsnano.6b07735.

(26) Arapov, K.; Rubingh, E.; Abbel, R.; Laven, J.; de With, G.; Friedrich, H. Conductive Screen Printing Inks by Gelation of Graphene Dispersions. Adv. Funct. Mater. 2016, 26 (4), 586-593.

https://doi.org/10.1002/adfm.201504030.

(27) Arapov, K.; Bex, G.; Hendriks, R.; Rubingh, E.; Abbel, R.; de With, G.; Friedrich, H. Conductivity Enhancement of Binder-Based Graphene Inks by Photonic Annealing and Subsequent Compression Rolling. Adv. Eng. Mater. 2016, 18 (7), 1234-1239. https://doi.org/10.1002/adem.201500646.

(28) Secor, E. B.; Gao, T. Z.; Islam, A. E.; Rao, R.; Wallace, S. G.; Zhu, J.; Putz, K. W.; Maruyama, B.; Hersam, M. C. Enhanced Conductivity, Adhesion, and Environmental Stability of Printed Graphene Inks with Nitrocellulose. Chem. Mater. 2017, 29 (5), 2332-2340. https://doi.org/10.1021/acs.chemmater.7b00029.

(29) Secor, E. B.; Gao, T. Z.; Dos Santos, M. H.; Wallace, S. G.; Putz, K. W.; Hersam, M. C. CombustionAssisted Photonic Annealing of Printable Graphene Inks via Exothermic Binders. ACS Appl. Mater. Interfaces 2017, 9 (35), 29418-29423. https://doi.org/10.1021/acsami.7b07189.

(30) Tobjörk, D.; Österbacka, R. Paper Electronics. Adv. Mater. 2011, 23 (17), 1935-1961. https://doi.org/10.1002/adma.201004692.

(31) Huang, X.; Leng, T.; Georgiou, T.; Abraham, J.; Raveendran Nair, R.; Novoselov, K. S.; Hu, Z. Graphene Oxide Dielectric Permittivity at GHz and Its Applications for Wireless Humidity Sensing. Sci. Rep. 2018, 8 (1), 43. https://doi.org/10.1038/s41598-017-16886-1.

(32) Seco, F.; Jiménez, A. R.; Seco, F.; Jiménez, A. R. Smartphone-Based Cooperative Indoor Localization with RFID Technology. Sensors 2018, 18 (1), 266. https://doi.org/10.3390/s18010266. 
(33) Bibi, F.; Guillaume, C.; Gontard, N.; Sorli, B. A Review: RFID Technology Having Sensing Aptitudes for Food Industry and Their Contribution to Tracking and Monitoring of Food Products. Trends Food Sci. Technol. 2017, 62, 91-103. https://doi.org/10.1016/J.TIFS.2017.01.013.

(34) Roscher, S.; Hoffmann, R.; Ambacher, O. Determination of the Graphene-graphite Ratio of Graphene Powder by Raman 2D Band Symmetry Analysis. Anal. Methods 2019, 11 (9), 1224-1228. https://doi.org/10.1039/C8AY02619J.

(35) Mori, F.; Kubouchi, M.; Arao, Y. Effect of Graphite Structures on the Productivity and Quality of FewLayer Graphene in Liquid-Phase Exfoliation. J. Mater. Sci. 2018, 53 (18), 12807-12815. https://doi.org/10.1007/s10853-018-2538-3.

(36) Wan, Y.-J.; Zhu, P.-L.; Yu, S.-H.; Sun, R.; Wong, C.-P.; Liao, W.-H. Graphene Paper for Exceptional EMI Shielding Performance Using Large-Sized Graphene Oxide Sheets and Doping Strategy. Carbon N. Y. 2017, 122, 74-81. https://doi.org/10.1016/J.CARBON.2017.06.042.

(37) Chen, J.; Li, Y.; Huang, L.; Jia, N.; Li, C.; Shi, G. Size Fractionation of Graphene Oxide Sheets via Filtration through Track-Etched Membranes. Adv. Mater. 2015, 27 (24), 3654-3660.

https://doi.org/10.1002/adma.201501271.

(38) Scidà, A.; Haque, S.; Treossi, E.; Robinson, A.; Smerzi, S.; Ravesi, S.; Borini, S.; Palermo, V. Application of Graphene-Based Flexible Antennas in Consumer Electronic Devices. Mater. Today 2018, 21 (3), 223 230. https://doi.org/10.1016/j.mattod.2018.01.007.

(39) Arao, Y.; Tanks, J. D.; Kubouchi, M.; Ito, A.; Hosoi, A.; Kawada, H. Direct Exfoliation of Layered Materials in Low-Boiling Point Solvents Using Weak Acid Salts. Carbon N. Y. 2019, 142, 261-268. https://doi.org/10.1016/J.CARBON.2018.10.063.

(40) Marrocco, G. The Art of UHF RFID Antenna Design: Impedance-Matching and Size-Reduction Techniques. IEEE Antennas Propag. Mag. 2008, 50 (1), 66-79. https://doi.org/10.1109/MAP.2008.4494504.

(41) Kim, T.; Kim, U.; Choi, J. Design of a Compact UHF RFID Tag Antenna Using an Inductively Coupled Parasitic Element. Microw. Opt. Technol. Lett. 2011, 53 (2), 239-242. https://doi.org/10.1002/mop.25753.

(42) Marrocco, G. RFID Antennas for the UHF Remote Monitoring of Human Subjects. IEEE Trans. Antennas Propag. 2007, 55 (6), 1862-1870. https://doi.org/10.1109/TAP.2007.898626.

(43) Rao, K. V. S.; Nikitin, P. V.; Lam, S. F. Antenna Design for UHF RFID Tags: A Review and a Practical Application. IEEE Trans. Antennas Propag. 2005, 53 (12), 3870-3876.

https://doi.org/10.1109/TAP.2005.859919.

(44) Fa-Shian Chang; Kin-Lu Wong; Tzung-Wern Chiou. Low-Cost Broadband Circularly Polarized Patch 
Antenna. IEEE Trans. Antennas Propag. 2003, 51 (10), 3006-3009.

https://doi.org/10.1109/TAP.2003.818010.

(45) Hazdra, P. Circular Polarization and Polarization Losses. 2006.

(46) Benjwal, P. Square Microstrip Antenna for Circular Polarization Operation; 2011; Vol. 36.

(47) Sharma, P.; Gupta, K. Analysis and Optimized Design of Single Feed Circularly Polarized Microstrip Antennas. IEEE Trans. Antennas Propag. 1983, 31 (6), 949-955.

https://doi.org/10.1109/TAP.1983.1143162.

(48) Bee Yen Toh; Cahill, R.; Fusco, V. F. Understanding and Measuring Circular Polarization. IEEE Trans. Educ. 2003, 46 (3), 313-318. https://doi.org/10.1109/TE.2003.813519.

(49) Pan, K.; Leng, T.; Zhou, X.; Ouslimani, H.; Hu, Z. Metamaterial Inspried Long Read Range UHF RFID Tag Antenna. In 2018 Progress in Electromagnetics Research Symposium (PIERS-Toyama); IEEE, 2018; pp 2192-2195. https://doi.org/10.23919/PIERS.2018.8598208.

(50) Su, W.; Cook, B. S.; Tentzeris, M. M. Additively Manufactured Microfluidics-Based "Peel-and-Replace" RF Sensors for Wearable Applications. IEEE Trans. Microw. Theory Tech. 2016, 64 (6), 1928-1936. https://doi.org/10.1109/TMTT.2016.2560177.

(51) Ha, U.; Ma, Y.; Zhong, Z.; Hsu, T.-M.; Adib, F. Learning Food Quality and Safety from Wireless Stickers. In Proceedings of the 17th ACM Workshop on Hot Topics in Networks - HotNets '18; ACM Press: New York, New York, USA, 2018; pp 106-112. https://doi.org/10.1145/3286062.3286078.

(52) Kaatze, U.; Behrends, R.; Pottel, R. Hydrogen Network Fluctuations and Dielectric Spectrometry of Liquids. J. Non. Cryst. Solids 2002, 305 (1-3), 19-28. https://doi.org/10.1016/S0022-3093(02)01084-0.

(53) Gregory, A. P.; Clarke, R. N.; (GB), N. P. L. C. for E. and T. M. (Great B. Tables of the Complex Permittivity of Dielectric Reference Liquids at Frequencies Up to $5 \mathrm{GHz}$; NPL report CETM; National Physical Laboratory, 2001.

(54) Caccami, M. C.; Manzari, S.; Marrocco, G. Phase-Oriented Sensing by Means of Loaded UHF RFID Tags. IEEE Trans. Antennas Propag. 2015, 63 (10), 4512-4520. https://doi.org/10.1109/TAP.2015.2465891.

(55) Nikitin, P. V.; Martinez, R.; Ramamurthy, S.; Leland, H.; Spiess, G.; Rao, K. V. S. Phase Based Spatial Identification of UHF RFID Tags. In 2010 IEEE International Conference on RFID (IEEE RFID 2010); IEEE, 2010; pp 102-109. https://doi.org/10.1109/RFID.2010.5467253.

(56) Jordan, M. I.; Mitchell, T. M. Machine Learning: Trends, Perspectives, and Prospects. Science 2015, 349 (6245), 255-260. https://doi.org/10.1126/science.aaa8415. 
(57) Romero, E.; Màrquez, L.; Carreras, X. Margin Maximization with Feed-Forward Neural Networks: A Comparative Study with SVM and AdaBoost. Neurocomputing 2004, 57, 313-344. https://doi.org/10.1016/J.NEUCOM.2003.10.011.

(58) Rumelhart, D. E.; Hinton, G. E.; Williams, R. J. Learning Representations by Back-Propagating Errors. Nature 1986, 323 (6088), 533-536. https://doi.org/10.1038/323533a0.

(59) Mathworks. Pattern recognition network - MATLAB patternnet - MathWorks United Kingdom https://uk.mathworks.com/help/deeplearning/ref/patternnet.html;jsessionid=69f371c03033e0491f1deeb406a 9 (accessed Mar 10, 2019).

(60) Schapire, R. E. Explaining AdaBoost. In Empirical Inference; Springer Berlin Heidelberg: Berlin, Heidelberg, 2013; pp 37-52. https://doi.org/10.1007/978-3-642-41136-6_5. 